\title{
Utility of 2-[18 F] fluoro-2-deoxy-D-glucose positron emission tomography in differential diagnosis of benign and malignant intraductal papillary-mucinous neoplasm of the pancreas
}

\author{
YOSHITO TOMIMARU ${ }^{1}$, YUTAKA TAKEDA ${ }^{1}$, MITSUAKI TATSUMI ${ }^{2}$, TONSOK KIM ${ }^{2}$, \\ SHOGO KOBAYASHI ${ }^{1}$, SHIGERU MARUBASHI ${ }^{1}$, HIDETOSHI EGUCHI $^{1}$, MASAHIRO TANEMURA $^{1}$, \\ TORU KITAGAWA ${ }^{1}$, HIROAKI NAGANO ${ }^{1}$, KOJI UMESHITA ${ }^{3}$, KENICHI WAKASA ${ }^{4}$ \\ YUICHIRO DOKI $^{1}$ and MASAKI MORI ${ }^{1}$ \\ Departments of ${ }^{1}$ Surgery, ${ }^{2}$ Radiology, and ${ }^{3}$ Division of Health Sciences, Graduate School of Medicine, \\ Osaka University, Suita, Osaka; ${ }^{4}$ Department of Pathology, Osaka City University Hospital, Osaka, Japan
}

Received April 1,2010; Accepted May 7, 2010

DOI: 10.3892/or_00000899

\begin{abstract}
Intraductal papillary-mucinous neoplasm (IPMN) of the pancreas presents in various histopathological stages from benign to malignant lesions. The differentiation between benign and malignant IPMN is important in order to determine the treatment of the patients. However, preoperative differentiation remains difficult. The aim of this study was to assess the utility of $2-\left[{ }^{18} \mathrm{~F}\right]$ fluoro-2-deoxy-Dglucose positron emission tomography (FDG-PET) in preoperative differentiation of benign and malignant IPMN of the pancreas. In the present study we prospectively investigated 29 patients who underwent CT, FDG-PET, and surgery for IPMNs, followed by histopathological examination. The maximum standardized uptake value (SUVmax) was determined on FDG-PET, and differentiation of benign from malignant IPMN was tested using various SUVmax cut-off levels and various parameters derived from the CT. SUVmax was found to be significantly higher in malignant IPMNs $(4.7 \pm 3.0)$ than that in benign IPMNs $(1.8 \pm 0.3$, $\mathrm{P}=0.0011)$. SUVmax values correlated with the histopathological types of IPMN (adenoma/borderline lesion/carcinoma in situ/invasive carcinoma) (Spearman rank correlation $0.865, \mathrm{P}<0.0001)$. The specificity, sensitivity and accuracy values were best for SUVmax of 2.5 (100, 93, and 96\%, respectively). The combination of mural nodule, detected on CT, and SUVmax of 2.5 offered the best diagnosis of malignant IPMN. These results suggest that FDG-PET is useful for differentiation of malignant IPMN of the pancreas,
\end{abstract}

Correspondence to: Dr Yutaka Takeda, Department of Surgery, Graduate School of Medicine, Osaka University, 2-2 Yamadaoka E-2, Suita, Osaka 565-0871 Japan

E-mail: ytakeda@gesurg.med.osaka-u.ac.jp

Key words: intraductal papillary-mucinous neoplasm, pancreas, positron emission tomography, standardized uptake value and that it should be performed in combination with other conventional imaging modalities.

\section{Introduction}

Intraductal papillary-mucinous neoplasm (IPMN) of the pancreas, which was first reported by Ohashi et al in 1982, originates from epithelial cells of the main pancreatic duct or its side branches and produces large amounts of mucin (1-4). IPMN presents at various histopathological stages from benign to malignant lesions, as classified by the WHO, including adenoma, borderline, carcinoma in situ (CIS), and invasive carcinoma $(5,6)$. While patients with benign IPMNs can be monitored without the need for surgery, malignant IPMNs should be resected surgically according to the grade of malignancy. Moreover, the postoperative prognosis of patients with invasive IPMNs is significantly poor and similar to that of patients with pancreatic ductal adenocarcinoma $(5,7,8)$. Therefore, preoperative differentiation between benign IPMN and malignant IPMN is important in order to determine the management of patients. To date, various features of malignant IPMN tumors using imaging techniques have been proposed, such as large lesion size, dilatation of the main pancreatic duct (MPD), and presence of mural nodules $(5,9-16)$. However, some of these features are controversial, and their accuracy depends on the imaging modalities used. Therefore, differentiation between benign and malignant IPMN is still difficult.

$2-\left[{ }^{18} \mathrm{~F}\right]$ fluoro-2-deoxy-D-glucose positron emission tomography (FDG-PET) is a sensitive and specific imaging protocol for the diagnosis and staging of several types of malignancies (17-20). To date, there have been few reports of FDG-PET in patients with IPMNs (21-25). Sperti et al (25) reported 47 cases with IPMNs confirmed histologically or cytologically, and concluded that FDG-PET was more accurate than conventional imaging techniques such as computed tomography (CT) and magnetic resonance imaging in distinguishing benign from malignant IPMN. In their report, however, the sensitivity, specificity, and accuracy of 


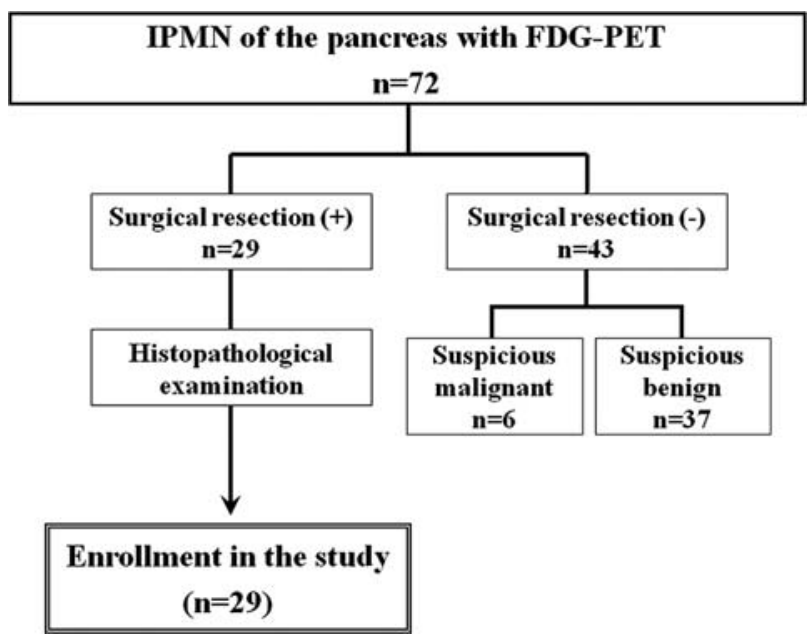

Figure 1. Distribution of the 72 patients with IPMN according to the histopathological examination. IPMN, intraductal papillary-mucinous neoplasm.

FDG-PET were evaluated only when the cut-off value of the maximum standardized uptake value (SUVmax) was set at 2.5. Moreover, although these figures were compared to those of whole CT findings, they were not compared to those of other radiological features reported to be associated with malignancy.

In the present study, by using the results of prospectively performed FDG-PET in patients with IPMN of the pancreas, we examined the correlation between the findings of FDGPET and the histopathological type of IPMN. Furthermore, we assessed usefulness of FDG-PET in differentiation between benign and malignant IPMN using several cut-off levels of SUVmax, and the utility of FDG-PET was compared to certain CT parameters and their combinations, in the diagnosis of malignant IPMN.

\section{Materials and methods}

Patients. Between January 2006 and June 2008, FDG-PET was prospectively performed in 72 patients with IPMN at Osaka University Hospital. In 29 patients out of the 72 patients, the tumor was resected surgically and then examined histopathologically. The surgically-resected 29 patients with histopathological confirmation of the IPMN were enrolled in the present study. The remaining 43 patients were decided to be followed up without surgical resection. The distribution of IPMN patients are shown in Fig. 1.

In principle, surgical resection of IPMN was scheduled for treatment only when the clinical features suggested malignancy. The features of tumors judged to be likely malignant on CT examination were IPMN with mural nodule, main duct type and combined type IPMN with $\geq 7 \mathrm{~mm}$ dilated MPD, combined type and branch type IPMN with $\geq 3-\mathrm{cm}$ cystic lesion, and histopathologically and/or cytologicallyconfirmed malignant IPMN. Among the 29 patients, 2 patients underwent surgery without fulfilling the above criterion; their clinical features were not suggestive of malignancy; one patient fervently desired resection of the IPMN and the other underwent IPMN resection at the same time as pancreatectomy for coexisting pancreatic ductal adeno-carcinoma. The type of selected surgical procedure performed was based on the location of IPMN. Pancreaticoduodenectomy was performed in 14 patients, distal pancreatectomy in 14 , and central pancreatectomy in the remaining one patient.

In the 43 patients without surgical resection, 6 patients, who had clinical features suggested malignancy, did not undergo surgery for the following reasons; poor risk at surgery in three patients, refusal to surgery in two patients, and concomitant liver metastasis in one patient. The remaining 37 patients without features suggested malignancy were followed up.

For all the patients, gender, age, clinical symptoms, tumor markers including carcinoembryonic antigen (CEA) and carbohydrate antigen 19-9 (CA19-9), tumor multiplicity, tumor location, IPMN type, diameter of cystic lesion, MPD dilatation, mural nodule, cytological diagnosis, histopathological diagnosis, and SUVmax of FDG-PET were prospectively investigated. Endoscopic retrograde cholangiopancreatography (ERCP) was performed for pancreatic duct lavage cytology and/or pancreatic juice cytology in 49 patients.

FDG-PET. Whole-body FDG-PET imaging was performed as described previously (26-28). Briefly, each patient fasted for at least $4 \mathrm{~h}$ before intravenous administration of $\sim 370 \mathrm{MBq}$ FDG. Serum glucose levels were determined just before FDG injection. Among the 72 patients, 70 patients were normoglycemic (blood glucose $<150 \mathrm{mg} / \mathrm{dl}$ ), and 2 patients were hyperglycemic (blood glucose $>220 \mathrm{mg} / \mathrm{ml}$ ). Simultaneous emission and transmission PET scans were acquired $1 \mathrm{~h}$ after FDG injection. Imaging was performed with a dedicated PET scanner (Headtome/Set 2400W; Shimadzu Co., Kyoto, Japan). Fusion images combined with PET images and CT images were composed using our previously described method (28). Since April 2007, FDG-PET/CT has been introduced to clinical practice in our hospital (FDG-PET and CT performed separately; n=30, FDG-PET/CT; n=42).

For semi-quantitative analysis, regions of interest were selected semi-automatically at the most intense area of FDG accumulation in the primary tumor on the PET image, and the SUVmax was calculated using the following formula: SUVmax $=$ PET count at most intense point $x$ calibration factor $(\mathrm{MBq} / \mathrm{kg}) /$ injection dose $(\mathrm{MBq}) /$ body weight $(\mathrm{kg})$.

In the absence of a visible FDG uptake, on the basis of the fusion images, regions of interest were drawn exactly on the area corresponding to the primary tumor, and the SUVmax was calculated.

The afore-mentioned 2 patients who were hyperglycemic at the PET examination contained one patient in the group of the patients with surgical resection, and one in the group of the patients without surgical resection. Since the SUVmax could not be calculated in these patients for the hyperglycemic state, they were excluded from the examination related to the SUVmax in this study.

CT. CT was performed either with a LightSpeed Qxi scanner (GE Medical Systems, Wis), a LightSpeed VCT scanner (GE Medical Systems) or an Aquilion 64 scanner (Toshiba Medical Systems, Japan) scanner using a tube voltage of 


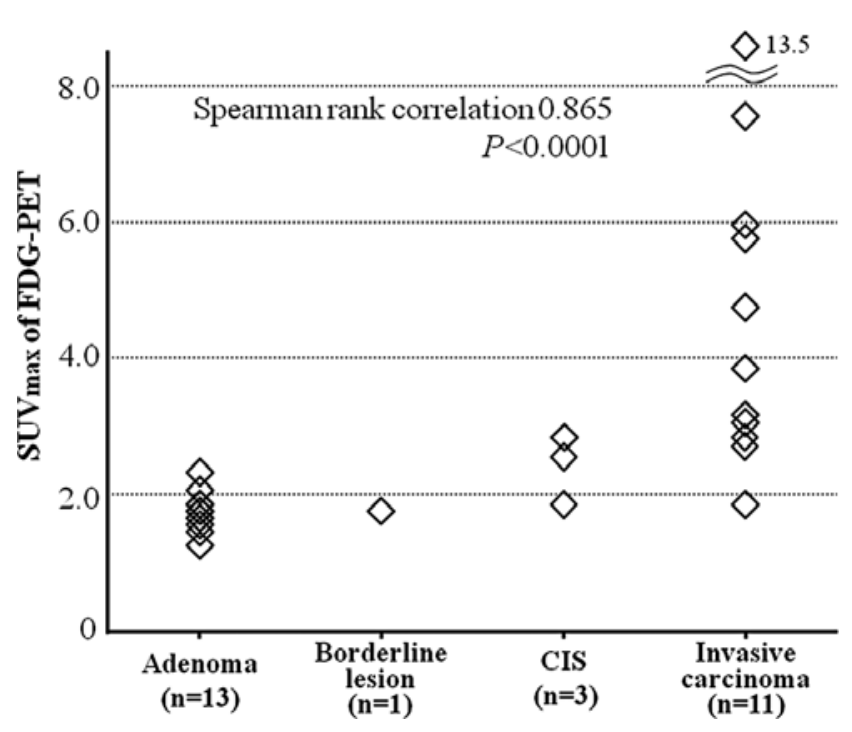

Figure 2. Correlation between histopathological type of IPMN and SUVmax of FDG-PET in 28 patients. Among the enrolled 29 patients, one patient with hyperglycemia at the FDG-PET examination was excluded. For abbreviations, see Fig. 1. SUVmax, maximum standardized uptake value; FDGPET, 2- $\left[{ }^{18} \mathrm{~F}\right]$ fluoro-2-deoxy-D-glucose positron emission tomography

$120 \mathrm{kV}$, a tube current of $300 \mathrm{~mA}$, and a rotation period of $0.5 \mathrm{sec}$. Images of $5 \mathrm{~mm}$ slice thickness were used for evaluation. Contrast-enhanced multiphasic CT images were acquired at $10 \mathrm{sec}$ after the peak aortic enhancement (arterial phase), followed by pancreatic phase and portal venous phase for upper abdomen. Nonionic contrast medium, $300 \mathrm{mg}$ of iodine per milliliter, was administered intravenously at a rate of $4 \mathrm{ml} / \mathrm{sec}$ with a power injector. Images were interpreted especially focusing on the presence of mural nodule as well as the size of tumor and presence of dilatation of main pancreatic duct.

Histopathological diagnosis of IPMN. The diagnosis of IPMN of the pancreas in the enrolled 29 patients was confirmed on histopathological examination of the resected specimens by an experienced pathologist. The lesions were histopathologically classified into the following subtypes: adenoma, borderline lesion, CIS, and invasive carcinoma. Adenoma and borderline lesions were categorized as benign lesions, while CIS and invasive carcinoma were categorized as malignant lesions.

Statistical analysis. Receiver operating characteristic (ROC) curve analysis was performed using sensitivity and specificity at various cut-off values. The significance of differences among the groups was assessed by the $\chi^{2}$, Fisher's exact test or the Mann-Whitney U test. Statistical analysis was performed using StatView (version 5.0, SAS Institute Inc, Cary, NC). A P-value $<0.05$ was considered statistically significant.

Ethical considerations. The study protocol was approved by the Human Ethics Review Committee of Osaka University Hospital and a signed consent form was obtained from each patient.

\section{Results}

Patient and tumor characteristics. Table I summarizes the clinical characteristics of the included 29 patients with histopathological confirmation. Among 27 patients who underwent ERCP, 9 were diagnosed as malignant IPMN based on pancreatic duct lavage cytology and/or pancreatic juice cytology. Five patients (17.2\%) had multiple cystic lesions. Five patients presented with high serum levels of CEA $(\geq 5.0 \mathrm{ng} / \mathrm{ml})$ and 5 patients with high CA 19-9 ( $\geq 37 \mathrm{U} / \mathrm{ml})$. The most common location of the lesion was in the head or uncinate of the pancreas $(44.8 \%)$. Three patients had the main duct type $(10.4 \%), 13$ the combined type (44.8\%), and 13 the branch type (44.8\%). Based on the CT findings, the mean diameter of the cystic lesion was $39 \mathrm{~mm}$ (range, 14-75 mm) in patients with combined type and branch type, and the MPD diameter was $11.1 \mathrm{~mm}$ (range, 5.0-41.0 mm) in patients with main duct type and combined type. Mural nodules were identified in 13 patients $(44.8 \%)$. Malignant IPMN was identified in $14(48.3 \%)$ patients, including 11 with invasive carcinoma and 3 with CIS. The remaining 15 patients $(51.7 \%)$ had benign IPMN: one borderline lesion, and 14 adenomas. The mean SUVmax of FDG-PET of the lesion for all patients was 3.3 (range, 1.3-13.5).

Correlation between histopathological type and SUVmax of FDG-PET. Fig. 2 displays the SUVmax for each histopathological type of IPMN. This analysis was performed in the 28 patients, while the remaining one patient with hyperglycemia at the FDG-PET examination was excluded from this analysis. The SUVmax correlated with the histopathological type (Spearman rank correlation 0.865, $\mathrm{P}<0.0001$ ). In detail, there were significant differences of the SUVmax between invasive carcinoma and others (CIS, borderline lesion, and adenoma), and between malignant IPMNs and benign IPMNs. Moreover, the SUVmax in patients with CIS was significantly higher than that with benign IPMNs. The following examination focuses on the difference between malignant IPMNs and benign IPMNs.

Comparison of clinical features of patients with benign and malignant IPMN. Table I summarizes the clinical features of patients with benign IPMNs and malignant IPMNs. There was no significant difference in gender, age, the presence of symptoms, serum levels of CEA and CA19-9, multiplicity, location, IPMN type, diameter of the lesion, and MPD diameter between the two groups. On the other hand, the frequency of the presence of mural nodule in malignant IPMN (92.9\%) was significantly higher than that in benign IPMN (20.0\%, P=0.0001). Furthermore, the SUVmax in patients with malignant IPMNs $(4.7 \pm 3.0)$ was significantly higher than that with benign IPMNs $(1.8 \pm 0.3, \mathrm{P}=0.0011)$.

Comparison of diagnosis of malignant IPMN by FDG-PET and $C T$. Table II lists the distribution of patients, sensitivity, specificity, and accuracy of FDG-PET and certain CT features of the tumors. Diagnosis of malignancy by FDG-PET was analyzed using various cut-off levels of SUVmax. Moreover, ROC curve was constructed by plotting sensitivity and specificity at various cut-off levels of SUVmax (Fig. 3). Such 
Table I. Clinical and histopathological characteristics of the patients with intraductal papillary-mucinous neoplasms (IPMN).

\begin{tabular}{|c|c|c|c|c|c|}
\hline & \multicolumn{3}{|c|}{ Histopathological confirmation (+) } & \multirow{2}{*}{$\begin{array}{c}\text { Histopathological } \\
\text { confirmation }(+) \\
(n=29)\end{array}$} & \multirow{2}{*}{$\begin{array}{c}\text { Histopathological } \\
\text { confirmation }(-) \\
(n=43)\end{array}$} \\
\hline & $\begin{array}{l}\text { Benign } \\
(n=15)\end{array}$ & $\begin{array}{l}\text { Malignant } \\
\quad(n=14)\end{array}$ & p-value & & \\
\hline Gender & & & 0.8367 & & \\
\hline Male & 8 & 8 & & 16 & 24 \\
\hline Female & 7 & 6 & & 13 & 19 \\
\hline Age (years) & $64 \pm 11$ & $66 \pm 9$ & 0.3736 & $65 \pm 10(48-82)$ & $68 \pm 11(41-90)$ \\
\hline Symptom & & & 0.1976 & & \\
\hline Present & 5 & 6 & & 11 & 2 \\
\hline Absent & 10 & 8 & & 18 & 41 \\
\hline CEA (ng/ml) & $2.1 \pm 1.0$ & $3.4 \pm 3.6$ & 0.1561 & $2.8 \pm 1.9(1-8)$ & $5.1 \pm 12.9(1-69)$ \\
\hline CA19-9 (U/ml) & $26.2 \pm 21.3$ & $38.3 \pm 50.1$ & 0.2118 & $28.3 \pm 48.3(5-240)$ & $22.1 \pm 14.7(5-60)$ \\
\hline Multiplicity & & & $>0.9999$ & & \\
\hline Solitary & 12 & 12 & & 24 & 28 \\
\hline Multiple & 3 & 2 & & 5 & 15 \\
\hline Location & & & 0.4865 & & \\
\hline Head or uncinate & 6 & 7 & & 13 & 19 \\
\hline Body & 4 & 5 & & 9 & 12 \\
\hline Tail & 5 & 2 & & 7 & 12 \\
\hline Type & & & 0.5859 & & \\
\hline Main duct & 1 & 2 & & 3 & 1 \\
\hline Combined & 6 & 7 & & 13 & 9 \\
\hline Branch duct & 8 & 5 & & 13 & 33 \\
\hline Diameter of cystic lesion $(\mathrm{mm})^{\mathrm{a}}$ & $41 \pm 15$ & $36 \pm 15$ & 0.3498 & $39 \pm 15(14-75)$ & $21 \pm 14(2-55)$ \\
\hline MPD diameter $(\mathrm{mm})^{\mathrm{b}}$ & $7.6 \pm 3.0$ & $13.8 \pm 11.9$ & 0.1993 & $11.1 \pm 9.5(5.0-41.0)$ & $4.8 \pm 3.0(2.1-11.0)$ \\
\hline Mural nodule & & & $>0.0001$ & & \\
\hline Present & 3 & 13 & & 16 & 6 \\
\hline Absent & 12 & 1 & & 13 & 37 \\
\hline Cytology ${ }^{c}$ & & & 0.0006 & & \\
\hline Positive & 0 & 9 & & 9 & 1 \\
\hline Negative & 13 & 5 & & 18 & 21 \\
\hline Histopathological type & & & - & & \\
\hline Adenoma & 14 & - & & 14 & - \\
\hline Borderline lesion & 1 & - & & 1 & - \\
\hline CIS & - & 3 & & 3 & - \\
\hline Invasive carcinoma & - & 11 & & 11 & - \\
\hline SUVmax of FDG-PET ${ }^{\mathrm{d}}$ & $1.8 \pm 0.3$ & $4.7 \pm 3.0$ & 0.0011 & $3.3 \pm 2.6(1.3-13.5)$ & $1.7 \pm 0.6(1.0-4.4)$ \\
\hline
\end{tabular}

${ }^{a}$ Diameter of cystic lesion was determined on CT in patients with combined and branch types. ${ }^{b}$ MPD diameter was determined on CT in patients with main duct and combined types. ${ }^{\circ}$ Cytology was performed in 49 patients. ${ }^{\text {d }}$ Two patients were excluded from analysis of SUVmax for hyperglycemia at the FDG-PET examination. CEA, carcinoembryonic antigen; CA19-9, carbohydrate antigen; MPD, main pancreatic duct; CIS, carcinoma in situ; SUVmax, maximum standardized uptake value; FDG-PET, 2-[ ${ }^{18}$ F] fluoro-2-deoxy-Dglucose positron emission tomography. 
Table II. Distribution of patients, sensitivity, specificity and accuracy of FDG-PET and certain CT parameters associated with a higher likelihood of malignancy.

\begin{tabular}{|c|c|c|c|c|c|c|c|c|c|c|c|c|c|}
\hline & & & & & & & & \multicolumn{6}{|c|}{ CT Findings } \\
\hline & & \multicolumn{6}{|c|}{ FDG-PET (SUVmax cut-off value) ${ }^{\mathrm{a}}$} & \multirow{2}{*}{\multicolumn{2}{|c|}{$\begin{array}{c}\text { Diameter } \\
\text { of cystic } \\
\text { lesion }(\mathrm{mm})^{\mathrm{b}}\end{array}$}} & \multirow{2}{*}{\multicolumn{2}{|c|}{$\begin{array}{c}\text { MPD } \\
\text { diameter } \\
(\mathrm{mm})^{\mathrm{c}}\end{array}$}} & \multirow{2}{*}{\multicolumn{2}{|c|}{$\begin{array}{c}\text { Mural } \\
\text { nodule }\end{array}$}} \\
\hline & & \multicolumn{2}{|c|}{2.0} & \multicolumn{2}{|c|}{2.5} & \multicolumn{2}{|c|}{3.0} & & & & & & \\
\hline & $\mathrm{n}$ & $(+)$ & $(-)$ & $(+)$ & $(-)$ & $(+)$ & $(-)$ & $\geq 30$ & $<30$ & $\geq 7$ & $<7$ & $(+)$ & $(-)$ \\
\hline Adenoma & 14 & 2 & 11 & 0 & 13 & 0 & 13 & 11 & 2 & 2 & 4 & 2 & 12 \\
\hline Borderline & 1 & 0 & 1 & 0 & 1 & 0 & 1 & 1 & 0 & 1 & 0 & 1 & 0 \\
\hline Carcinoma in situ & 3 & 2 & 1 & 2 & 1 & 0 & 3 & 1 & 1 & 1 & 2 & 2 & 1 \\
\hline Invasive carcinoma & 11 & 11 & 0 & 11 & 0 & 10 & 1 & 6 & 4 & 5 & 1 & 11 & 0 \\
\hline Sensitivity (\%) & & \multicolumn{2}{|c|}{93} & \multicolumn{2}{|c|}{93} & \multicolumn{2}{|c|}{71} & \multicolumn{2}{|c|}{58} & \multicolumn{2}{|c|}{67} & \multicolumn{2}{|c|}{93} \\
\hline Specificity (\%) & & \multicolumn{2}{|c|}{86} & \multicolumn{2}{|c|}{100} & \multicolumn{2}{|c|}{100} & \multicolumn{2}{|c|}{14} & \multicolumn{2}{|c|}{57} & \multicolumn{2}{|c|}{80} \\
\hline Accuracy $(\%)$ & & \multicolumn{2}{|c|}{89} & \multicolumn{2}{|c|}{96} & \multicolumn{2}{|c|}{86} & \multicolumn{2}{|c|}{35} & \multicolumn{2}{|c|}{63} & \multicolumn{2}{|c|}{86} \\
\hline
\end{tabular}

${ }^{a}$ In the analysis of the FDG-PET (SUVmax cut-off value) (left side), one adenoma patient with hyperglycemia at the FDG-PET examination was excluded. biameter of cystic lesion was determined on CT in patients with combined and branch types. ${ }^{\mathrm{c}} \mathrm{MPD}$ diameter was determined on CT in patients with main duct and combined types. FDG-PET, 2- $\left[{ }^{18} \mathrm{~F}\right]$ fluoro-2-deoxy-D-glucose positron emission tomography; SUVmax, maximum standardized uptake value; MPD, main pancreatic duct.

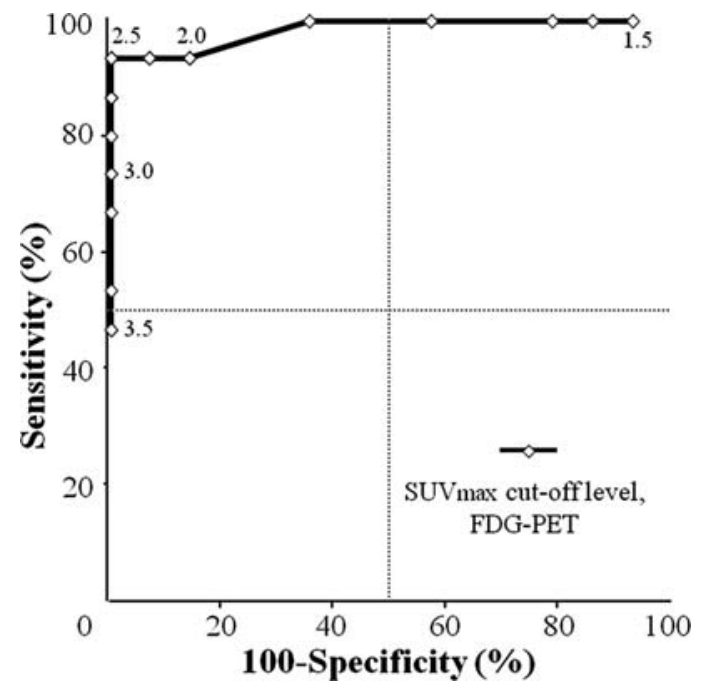

Figure 3. ROC curve for the differential diagnosis of benign IPMN and malignant IPMN according to SUVmax of FDG-PET. Data on the curve represent SUVmax cut-off levels of 1.5-3.5. For abbreviations, see Fig. 1. ROC, receiver operating characteristic.

100 , and $96 \%$, respectively. These values were superior to those of CT parameters that had been reported previously to be associated with a higher likelihood of malignancy $(5,9-17)$, including large cystic lesion size $(\geq 3.0 \mathrm{vs} .<3.0 \mathrm{~cm})$, and large dilatation of MPD ( $\geq 7$ vs. $<7 \mathrm{~mm}$ ) (Table II). In comparison to the presence/absence of mural nodules, which is also previously reported to be associated with malignancy, though the sensitivity was almost equal, the specificity and accuracy were superior (Table II).

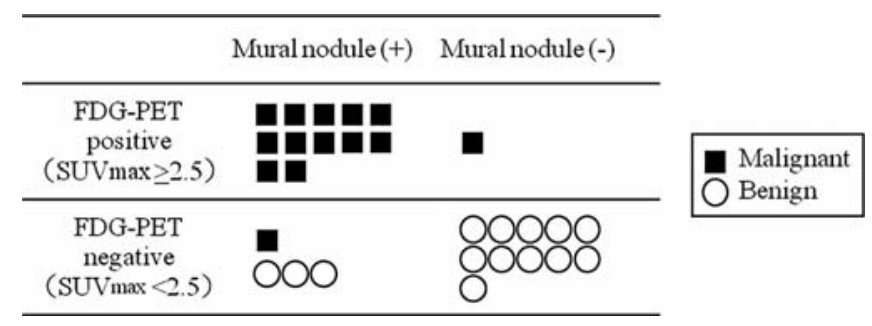

Figure 4. Correlation between histopathological type and results of combination of FDG-PET (SUVmax) and CT (mural nodule) in 28 patients with IPMNs. Among the enrolled 29 patients, one patient with hyperglycemia at the FDG-PET examination was excluded. For abbreviations, see Fig. 1. CT, computed tomography.

Combination of FDG-PET findings and mural nodule in diagnosis of malignancy. Based on the above results, both the FDG-PET findings and the presence/absence of mural nodule on $\mathrm{CT}$ were considered useful in differentiation of malignant IPMN. Therefore, the combination of SUVmax of FDG-PET and mural nodule on CT were used for the diagnosis of malignancy (Fig. 4). In this analysis, SUVmax of $\geq 2.5$ was considered positive for malignancy. All patients with SUVmax $\geq 2.5$ and mural nodule were histopathologically confirmed to have malignant IPMN $(n=12)$, and all patients with SUVmax $<2.5$ and no mural nodule had benign IPMN $(n=11)$. A representative patient with malignant IPMN, positive PET finding and mural nodule is shown in Fig. 5A. In the remaining 5 patients, there was discrepancy in PET findings and presence/absence of mural nodule. Four of the 5 patients had mural nodule on CT scans, but their nodule was negative on FDG-PET. Among the 4 patients, 3 were confirmed to have benign tumors and one had malignant IPMN. 

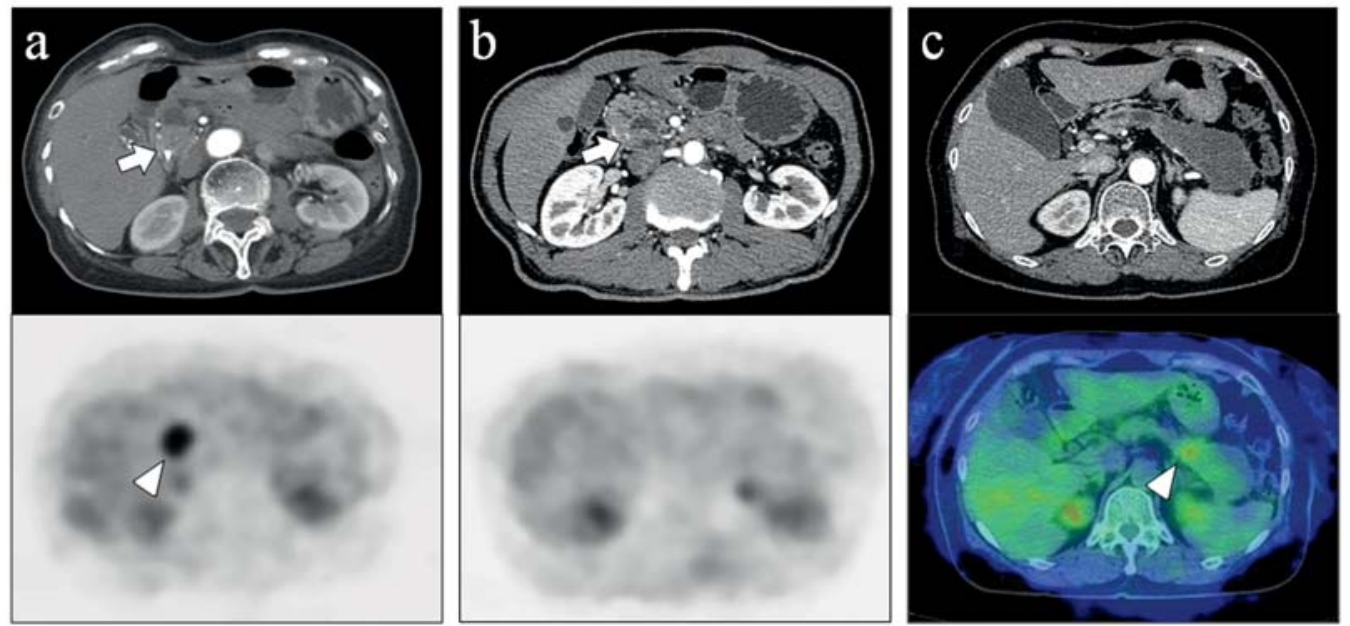

Figure 5. CT and FDG-PET findings of three representative cases [(A) malignant IPMN, (B) benign IPMN, (C) malignant IPMN]. Arrows, mural nodules on CT; arrowheads, the most intense area of FDG accumulation on PET. For abbreviations, see Fig. 1. CT, computed tomography.

The CT and FDG-PET of a representative patient with mural nodule who had negative FDG-PET is shown in Fig. 5B. On the other hand, one patient with malignant IPMN had a positive lesion on FDG-PET (in pancreatic body), but mural nodule could not be identified even on thin-slice CT scans (Fig. 5C).

Follow-up of patients without surgical resection. The clinical characteristics of the 43 patients followed up without surgical resection are also shown in Table I. In 4 of the 43 patients followed up, the SUVmax was $\geq 2.5$. One patient, who had a mural nodule suggesting malignancy with the SUVmax being 4.4, did not undergo surgery because of refusal, but wished to be followed up without any treatment. Now, 10 months after the start of follow-up, the tumor has been gradually increasing and a pulmonary metastasis has identified. In one patient having a mural nodule with the SUVmax being 3.2, who also did not undergo surgery for poor risk and followed-up without any treatment, the tumor has been gradually increasing, now, 24 months after the start of follow-up. In the remaining 2 patients, the tumor had gradually progressed despite chemotherapy, and then they died of the invasive cancer. On the other hand, in the remaining 39 patients with the SUVmax $<2.5$, the tumor has been unchanged for the follow-up period. The median follow-up period of the 39 patients was 24 months. Under the condition that the histopathological type of IPMN in the patients with growing tumor and the patients with unchanged tumor were hypothesized as malignant and benign, respectively, the sensitivity, specificity, and accuracy of FDG-PET with cut-off level being 2.5 for the differentiation of malignant IPMN were 100, 100, and $100 \%$.

\section{Discussion}

In the present study, the sensitivity, specificity, and accuracy of FDG-PET were examined using several cut-off levels of SUVmax to differentiate between benign and malignant IPMN of the pancreas. The results showed that at the optimal cut-off level of SUVmax was 2.5, with the specificity, sensitivity and accuracy being 93, 100 and 96\%, respectively. Mansour et al (24) previously examined 68 patients with pancreatic cystic tumors and reported that the sensitivity and specificity of PET for malignancy were 57 and $85 \%$, respectively. However, only 5 patients had an IPMN, and since detailed criteria for the positive/negative PET findings were not reported in that study, it is difficult to compare the results of our current study to their study. On the other hand, Sperti et al (25) reported that FDG-PET was useful for differentiation of benign and malignant IPMN with the specificity, sensitivity and accuracy of 92, 97 and 95\%, respectively, using a cut-off level of SUVmax of 2.5, though it was not clear in their report whether they had investigated the usefulness with any cut-off levels of SUVmax other than 2.5. The results of the present study were in agreement with those reported by Sperti et al (25). However, since such sensitivity, specificity, and accuracy calculations generally depend highly on the underlying prevalence of the disease, one should also consider the underlying prevalence of the diseases, in the comparison of such calculations.

In the use of FDG-PET imaging for the diagnosis of pancreatic tumors, we should remember that the FDG can accumulate in chronic and acute pancreatitis and results in false-positive interpretations on PET imaging. Furthermore, IPMN is sometimes associated with secondary pancreatitis due to disturbance in the run-off of pancreatic juice (29). However, in the present study, the specificity of FDG-PET was $100 \%$, and false-positive PET scans from pancreatitis were not observed.

In addition to the FDG-PET findings, we also examined in the present study the diagnostic utility of conventional modalities including pancreatic duct lavage cytology and/or pancreatic juice cytology and certain imaging parameters, which are known to be associated with a higher likelihood of malignancy. The results of pancreatic duct lavage cytology and/or pancreatic juice cytology also actually helped discriminate benign and malignant tumors in this study. However, cytology frequently results in false-negatives or inconclusive results, and it is of diagnostic value only if cancer is proven. Therefore, it is difficult to compare its usefulness 
to that of other modalities. This study also showed the low power of other imaging parameters, apart from mural nodule, such as large/small size of cystic lesion and dilated/nondilated MPD, in the differentiation of malignant from benign IPMN, which may be partly due to the potential prejudices in selection of the enrolled patients; the enrolled patients might be necessary for surgery. On the other hand, the presence/ absence of mural nodule, which has been considered an indicator of malignancy in IPMN, was found to be useful in the differentiation of malignancy in this study $(12,13,15-17)$. The sensitivity, specificity, and accuracy of the presence/ absence of mural nodule in the differentiation of malignant IPMN were 93, 80 and $86 \%$, respectively. This diagnostic value of the presence/absence of mural nodule was in agreement with some previous reports $(15,16)$.

In this analysis, the specificity and accuracy of the presence/absence of mural nodule in the differentiation of malignant IPMN were just slightly inferior to those of the FDG-PET findings, and the sensitivity was almost equal, which suggests that we can actually obtain information enough for the differentiation from CT findings alone. However, as shown in Table II and Fig. 4, there were four cases with discrepancy between the presence/absence of mural nodule and histopathological finding (one malignant patient positive on FDG-PET without mural nodule, and three benign patients with mural nodule and negative FDG-PET finding). In all the four cases, the FDG-PET findings with SUVmax cut-off value being 2.5 , corresponded perfectly to the histopathological findings. Considering such clinical significance in the FDG-PET, the FDG-PET examination was found significant in combination with CT. Reflecting the result of this study, patients with positive FDG-PET finding despite negative CT findings should be considered as potentially malignant, and be recommended undergoing surgery. On the other hand, now, we cannot exclude PET-negative patients with suspicious malignant CT finding from surgical indication. However, in future, by collecting histopathological information of such patients, some of such patients may be expected to be followed-up without surgical resection.

In addition to the surgically-resected IPMN patients, IPMN patients without surgical resection were investigated. In general, since most IPMNs are generally slow-growing tumors, it is not determined that IPMNs which have been unchanged for a certain period without histopathological confirmation of the resected specimens are considered to be benign (30). For this reason, patients without histopathological confirmation of the resected specimens were excluded from the present study, and only patients with IPMN which had been histopathologically confirmed in resected specimens were included in the present study. However, in the patients without surgical resection, the tumor with the SUVmax $<2.5$ has been unchanged during the follow-up period, and the tumor with the SUVmax $\geq 2.5$ had grown, which suggests association of tumor progression with the FDG-PET findings. Considering the association, the utility of the FDG-PET in the differentiation of benign and malignant IPMN is suggested to be validated in patients without surgical resection.

In summary, FDG-PET is a useful imaging technique for the diagnosis of malignant IPMN of the pancreas when used with SUVmax cut-off level of 2.5, with specificity, sensitivity and accuracy of 93, 100 and 96\%, respectively. We recommend the use of FDG-PET in combination with $\mathrm{CT}$ for the diagnosis of malignant IPMN of the pancreas.

\section{References}

1. Ohashi K, Murakami Y and Murayama M: Four cases of mucus secreting pancreatic cancer. Prog Dig Endosc 20: 348-351, 19982.

2. Sugiyama M and Atomi Y: Intraductal papillary mucinous tumors of the pancreas: imaging studies and treatment strategies. Ann Surg 228: 685-691, 1998.

3. Sohn TA, Yeo CJ, Cameron JL, Iacobuzio-Donahue CA, Hruban RH and Lillemoe KD: Intraductal papillary mucinous neoplasms of the pancreas: an increasingly recognized clinicopathologic entity. Ann Surg 234: 313-321, 2001.

4. Suzuki Y, Atomi Y, Sugiyama M, et al: Japanese multiinstitutional study of intraductal papillary mucinous tumor and mucinous cystic tumor. Cystic neoplasm of the pancreas: a Japanese multiinstitutional study of intraductal papillary mucinous tumor and mucinous cystic tumor. Pancreas 28: 241-246, 2004

5. Yamaguchi K, Ogawa Y, Chijiiwa K and Tanaka M: Mucinhypersecreting tumors of the pancreas: assessing the grade of malignancy preoperatively. Am J Surg 171: 427-431, 1996.

6. Kloppel G, Solcia E, Longnecker DS, Capella C and Sobin LH: Histological typing of tumours of the exocrine pancreas. In: World Health Organization. International Histological Classification of Tumors. 2nd edition. Springer, Berlin, pp11-20, 1996.

7. Yamao K, Ohashi K, Nakamura T, et al: The prognosis of intraductal papillary mucinous tumors of the pancreas. Hepatogastroenterology 47: 1129-1134, 2000.

8. Salvia R, Fernández-del Castillo C, Bassi C, et al: Main-duct intraductal papillary mucinous neoplasms of the pancreas: clinical predictors of malignancy and long-term survival following resection. Ann Surg 239: 678-685, 2004.

9. Cellier C, Cuillerier E, Palazzo L, et al: Intraductal papillary and mucinous tumors of the pancreas: accuracy of preoperative computed tomography, endoscopic retrograde pancreatography and endoscopic ultrasonography, and long-term outcome in a large surgical series. Gastrointest Endosc 47: 42-49, 1998.

10. Bernard P, Scoazec JY, Joubert M, Kahn X, Le Borgne J, Berger $\mathrm{F}$ and Partensky $\mathrm{C}$ : Intraductal papillary-mucinous tumors of the pancreas: predictive criteria of malignancy according to pathological examination of 53 cases. Arch Surg 137: 1274-1278, 2002.

11. Sugiyama M, Izumisato $\mathrm{Y}$, Abe $\mathrm{N}$, Masaki $\mathrm{T}$, Mori $\mathrm{T}$ and Atomi Y: Predictive factors for malignancy in intraductal papillary-mucinous tumours of the pancreas. Br J Surg 90: 1244-1249, 2003.

12. Baba $\mathrm{T}$, Yamaguchi $\mathrm{T}$, Ishihara $\mathrm{T}$, et al: Distinguishing benign from malignant intraductal papillary mucinous tumors of the pancreas by imaging techniques. Pancreas 29: 212-217, 2004.

13. Itoh T, Hirooka Y, Itoh A, et al: Usefulness of contrastenhanced transabdominal ultrasonography in the diagnosis of intraductal papillary mucinous tumors of the pancreas. Am J Gastroenterol 100: 144-152, 2005.

14. Jang JY, Kim SW, Ahn YJ, et al: Multicenter analysis of clinicopathologic features of intraductal papillary mucinous tumor of the pancreas: is it possible to predict the malignancy before surgery? Ann Surg Oncol 12: 124-132, 2005.

15. Chiu SS, Lim JH, Lee WJ, et al: Intraductal papillary mucinous tumour of the pancreas: differentiation of malignancy and benignancy by CT. Clin Radiol 61: 776-783, 2006.

16. Takeshita K, Kutomi K, Takada K, et al: Differential diagnosis of benign or malignant intraductal papillary mucinous neoplasm of the pancreas by multidetector row helical computed tomography: evaluation of predictive factors by logistic regression analysis. J Comput Assist Tomogr 32: 191-197, 2008.

17. Nakamoto Y, Higashi T, Sakahara H, et al: Delayed (18)Ffluoro-2-deoxy-D-glucose positron emission tomography scan for differentiation between malignant and benign lesions in the pancreas. Cancer 89: 2547-2554, 2000.

18. Pakzad F, Groves AM and Ell PJ: The role of positron emission tomography in the management of pancreatic cancer. Semin Nucl Med 36: 248-256, 2006. 
19. Chen YK, Su CT, Chi KH, Cheng RH, Wang SC and Hsu CH: Utility of 18F-FDG PET/CT uptake patterns in Waldeyer's ring for differentiating benign from malignant lesions in lateral pharyngeal recess of nasopharynx. J Nucl Med 48: 8-14, 2007.

20. Fletcher JW, Kymes SM, Gould M, et al: VA SNAP Cooperative Studies Group. A comparison of the diagnostic accuracy of 18F-FDG PET and CT in the characterization of solitary pulmonary nodules. J Nucl Med 49: 179-185, 2008.

21. Sperti C, Pasquali C, Chierichetti F, Liessi G, Ferlin G and Pedrazzoli S: Value of 18-fluorodeoxyglucose positron emission tomography in the management of patients with cystic tumors of the pancreas. Ann Surg 234: 675-680, 2001.

22. Yoshioka M, Sato T, Furuya T, et al: Positron emission tomography with 2-deoxy-2-[(18)F] fluoro-d-glucose for diagnosis of intraductal papillary mucinous tumor of the pancreas with parenchymal invasion. J Gastroenterol 38: 1189-1193, 2003.

23. Sperti C, Pasquali C, Decet G, Chierichetti F, Liessi G and Pedrazzoli S: F-18-fluorodeoxyglucose positron emission tomography in differentiating malignant from benign pancreatic cysts: a prospective study. J Gastrointest Surg 9: 22-28, 2005.

24. Mansour JC, Schwartz L, Pandit-Taskar N, et al: The utility of F-18 fluorodeoxyglucose whole body PET imaging for determining malignancy in cystic lesions of the pancreas. J Gastrointest Surg 10: 1354-1360, 2006.
25. Sperti C, Bissoli S, Pasquali C, Frison L, Liessi G, Chierichetti F and Pedrazzoli S: 18-fluorodeoxyglucose positron emission tomography enhances computed tomography diagnosis of malignant intraductal papillary mucinous neoplasms of the pancreas. Ann Surg 246: 932-937, 2007.

26. Paul AK, Tatsumi M, Higuchi I, Fukunaga H, Yasuda T and Nishimura T: Gamma camera coincidence imaging with $\left[{ }^{18} \mathrm{~F}\right]$ fluorodeoxyglucose in the pretreatment evaluation of patients with oesophageal cancer. Nucl Med Commun 24: 963-970, 2003.

27. Tatsumi M, Yutani K, Watanabe Y, et al: Feasibility of fluorodeoxyglucose dual-head gamma camera coincidence imaging in the evaluation of lung cancer: comparison with FDG PET. J Nucl Med 40: 566-573, 1999.

28. Fukunaga H, Sekimoto M, Ikeda M, et al: Fusion image of positron emission tomography and computed tomography for the diagnosis of local recurrence of rectal cancer. Ann Surg Oncol 12: 561-569, 2005.

29. Goh BK, Tan YM, Cheow PC, Chung YF, Chow PK, Wong WK and Ooi LL: Cystic neoplasms of the pancreas with mucinproduction. Eur J Surg Oncol 31: 282-287, 2005.

30. Tanaka M: Intraductal papillary mucinous neoplasm of the pancreas: diagnosis and treatment. Pancreas 28: 282-288, 2004. 